Commun. Korean Math. Soc. 29 (2014), No. 3, pp. 415-420

http://dx.doi.org/10.4134/CKMS.2014.29.3.415

\title{
DIRICHLET PROBLEM FOR POLYNOMIALS ON THE UNIT DISK
}

\author{
Ali Sinan Sertöz
}

ABstract. We give explicit formulas, without using the Poisson integral, for the functions that are C-harmonic on the unit disk and restrict to a prescribed polynomial on the boundary.

\section{Introduction}

It is known that the special Dirichlet problem in $\mathbb{R}^{2}$, which asks if there is a harmonic function on the unit disc that extends continuously on the boundary to a given continuous function has an affirmative answer via the Poisson integral. (Such functions are called C-harmonic.)

It is however surprising that an explicit and highly symmetric formula exists for the solution of the Dirichlet problem on the unit disc for polynomials, and it is this formula that we share in this paper.

A concise summary of what is known in this direction is as follows. It is known for example that if the boundary condition is a polynomial, then the solution function is also a polynomial, see [1, Theorem 5.1]. Moreover in [4] the unit circle is replaced by some real algebraic curve and the polynomial solutions to the Dirichlet problem are classified in this new set-up. When the disc is replaced by a connected bounded domain in the plane, the same problem, this time with rational boundary data, is discussed explicitly in [5]. Further, in $[2,3]$, a characterization of the disc is given as the only domain where the Dirichlet problem can be solved by certain algebraic functions. For some of the the higher dimensional aspects of the theory we refer to [6].

The bases for harmonic polynomials is well known in the literature but for the explicit determination of the coefficients the literature gives only algorithms, see for example the appendix in [1]. Here we show that these coefficients can be analytically determined, see Theorem 2.1. The required coefficients are closely related to expansions in terms of Chebyshev polynomials.

Received January 30, 2014.

2010 Mathematics Subject Classification. Primary 31A25; Secondary 31A05.

Key words and phrases. harmonic polynomials, Dirichlet problem. 


\section{Main results}

We let $\Omega=\{z \in \mathbb{C}|| z \mid<1\}$ denote the unit disc, where $z=x+i y$ is the complex variable as usual. Define $C H(\Omega)$ to be the set of functions which are continuous on the closed disc $\bar{\Omega}$ and are harmonic on $\Omega$. Let $\partial \Omega$ be the unit circle $|z|=1$.

Given a polynomial $P_{N}(x, y)$ of degree $N \geq 0$, we want to find a function $F(x, y) \in C H(\Omega)$ such that $\left.F(x, y)\right|_{\partial \Omega}=\left.P_{N}(x, y)\right|_{\partial \Omega}$. Since $y^{2}=1-x^{2}$ on $\partial \Omega$, it suffices to take

$$
P_{N}(x, y)=\alpha_{0}+\sum_{i=1}^{N}\left(\alpha_{i} x^{i}+\beta_{i} y x^{i-1}\right),
$$

where $\alpha_{i}$ and $\beta_{i}$ are complex constants. Suppose now that there exist functions $u_{n}(x, y), v_{n+1}(x, y) \in C H(\Omega)$ such that

$$
\left.u_{n}(x, y)\right|_{\partial \Omega}=x^{n} \text { and }\left.v_{n+1}(x, y)\right|_{\partial \Omega}=y x^{n}, \quad n=0,1, \ldots
$$

Then the unique solution to the above Dirichlet problem is given by

$$
F(x, y)=\alpha_{0}+\sum_{i=1}^{N}\left(\alpha_{i} u_{i}(x, y)+\beta_{i} v_{i}(x, y)\right) .
$$

The usual Poisson integral determines $u_{n}(x, y)$ and $v_{n}(x, y)$ as

$$
u_{n}(r \cos t, r \sin t)=\frac{1}{2 \pi} \int_{0}^{2 \pi} \frac{\cos ^{n} \theta\left(1-r^{2}\right)}{1-2 \cos (\theta-t)+r^{2}} d \theta,
$$

and

$$
v_{n+1}(r \cos t, r \sin t)=\frac{1}{2 \pi} \int_{0}^{2 \pi} \frac{\cos ^{n} \theta \sin \theta\left(1-r^{2}\right)}{1-2 \cos (\theta-t)+r^{2}} d \theta,
$$

where $|r|<1$ and $n=0,1, \ldots$.

While absolutely correct, these integrals are formidable to evaluate explicitly. Instead we will construct $u_{n}(x, y)$ and $v_{n}(x, y)$ directly from the real and imaginary parts of $z^{k}, k=0,1, \ldots, n$ as follows. First let

$$
\varphi_{k}(x, y)= \begin{cases}\operatorname{Re}(x+i y)^{k} & \text { if } k \geq 1 \\ 1 & \text { if } k=0 \\ 0 & \text { if } k<0\end{cases}
$$

and

$$
\psi_{k}(x, y)= \begin{cases}\operatorname{Im}(x+i y)^{k} & \text { if } k \geq 1 \\ 0 & \text { if } k \leq 0\end{cases}
$$

Clearly each $\varphi_{k}(x, y)$ and $\psi_{k}(x, y)$ is harmonic on the whole plane, being the real or imaginary part of the entire function $(x+i y)^{k}$.

We can now state the explicit expressions of $u_{n}(x, y)$ and $v_{n}(x, y)$ as linear combinations of $\varphi_{k}(x, y)$ and $\psi_{k}(x, y)$. Clearly

$$
u_{0}(x, y)=1, u_{1}(x, y)=x, v_{1}(x, y)=y \text {, and } v_{2}(x, y)=x y \text {. }
$$


In general we have the following theorem.

Theorem 2.1. For each $n \geq 0$, the unique functions $u_{n}(x, y)$ and $v_{n}(x, y)$ in $C H(\Omega)$ satisfying

$$
\left.u_{n}(x, y)\right|_{\partial \Omega}=x^{n}, \text { and }\left.v_{n+1}(x, y)\right|_{\partial \Omega}=y x^{n}
$$

are given as, for $n \geq 0$,

$$
u_{n}(x, y)=\frac{1}{2^{n-1}} \sum_{k=0}^{\left\lfloor\frac{n-1}{2}\right\rfloor}\left(\begin{array}{c}
n-1 \\
k
\end{array}\right)\left(\varphi_{n-2 k}(x, y)+\varphi_{n-2 k-2}(x, y)\right),
$$

and for $n \geq 1$,

$$
v_{n}(x, y)=\frac{1}{2^{n-1}} \sum_{k=0}^{\left\lfloor\frac{n-1}{2}\right\rfloor}\left(\begin{array}{c}
n-1 \\
k
\end{array}\right)\left(\psi_{n-2 k}(x, y)-\psi_{n-2 k-2}(x, y)\right) .
$$

Note that the statements of the above theorem are equivalent to the following equalities which will be more convenient to refer to during the proof. Equation (2.1) is equivalent to

$$
u_{n}(x, y)=\frac{1}{2^{n-1}} \sum_{k=0}^{\left\lfloor\frac{n}{2}\right\rfloor}\left(\begin{array}{l}
n \\
k
\end{array}\right) \varphi_{n-2 k}(x, y)-\frac{\epsilon_{n}}{2^{n}}\left(\begin{array}{c}
n \\
\left\lfloor\frac{n}{2}\right\rfloor
\end{array}\right),
$$

where $n=0,1, \ldots$, and

$$
\epsilon_{n}= \begin{cases}1 & \text { if } n \text { is even, } \\ 0 & \text { if } n \text { is odd. }\end{cases}
$$

For $v_{n}(x, y)$, Equation (2.2) is equivalent to

$$
v_{n}(x, y)=\frac{1}{2^{n-1}} \sum_{k=0}^{\left\lfloor\frac{n}{2}\right\rfloor} \frac{n-2 k}{n}\left(\begin{array}{l}
n \\
k
\end{array}\right) \psi_{n-2 k}(x, y),
$$

where $n=1,2, \ldots$

\section{Chebyshev polynomials}

In this section we remind some basic facts about Chebyshev polynomials of the first and second kinds which we will use in the next section. For details we refer to two sources [7] and [8], one classical and one recent.

Since each $\psi_{k}(x, y)$ is divisible by $y$, we define a new polynomial $\widetilde{\psi}_{\ell}(x, y)$ by the relation

$$
\psi_{k}(x, y)=\widetilde{\psi}_{k-1}(x, y) y, \quad k=0,1, \ldots
$$

Moreover since the power of $y$ in each monomial of the polynomials $\varphi_{k}(x, y)$ and $\widetilde{\psi}_{k}(x, y)$ is even, by restricting these polynomials to the unit circle $|z|=1$ by putting $y^{2}=1-x^{2}$, we obtain two types of polynomials of $x$ as follows.

$$
T_{k}(x):=\varphi_{k}\left(x, \sqrt{1-x^{2}}\right), \quad k=0,1, \ldots,
$$


and

$$
U_{k}(x):=\widetilde{\psi}_{k}\left(x, \sqrt{1-x^{2}}\right), \quad k=-1,0, \ldots
$$

Using the obvious relations

$$
\varphi_{n+1}(x, y)=x \varphi_{n}(x, y)-y \psi_{n}(x, y)
$$

and

we obtain

$$
\psi_{n+1}(x, y)=x \psi_{n}(x, y)+y \varphi_{n}(x, y)
$$

and

$$
\varphi_{n+1}(x, y)=x \varphi_{n}(x, y)-y^{2} \widetilde{\psi}_{n-1}(x, y)
$$

$$
\widetilde{\psi}_{n}(x, y)=x \widetilde{\psi}_{n-1}(x, y)+\varphi_{n}(x, y) .
$$

Restricting these equations to the unit circle by putting $y^{2}=1-x^{2}$ we get

$$
T_{n+1}(x)=x T_{n}(x)-\left(1-x^{2}\right) U_{n-1}(x)
$$

and

$$
U_{n}(x)=x U_{n-1}(x)+T_{n}(x) .
$$

Since we already have $T_{0}(x)=1$ and $U_{-1}(x)=0$, we conclude that $T_{n}(x)$ and $U_{n}(x)$ are the Chebyshev polynomials of the first and second kinds, respectively.

Chebyshev polynomials of the first and second kinds are related to each other by the identity

$$
\frac{d}{d x} T_{n}(x)=n U_{n-1}(x) \text { for } n=0,1, \ldots
$$

\section{Proof of the main theorem}

In this section we prove Theorem 2.1.

We start by noting that the leading monomial in $T_{n}(x)$ is $x^{n}$, and only monomials of the form $x^{n-2 k}$ appear with a non-zero coefficient in the expression of $T_{n}(x)$, where $0 \leq k \leq\left\lfloor\frac{n}{2}\right\rfloor$. Therefore we can find constants $c_{n-2 k}^{(n)}$ such that

$$
x^{n}=\sum_{k=0}^{\left\lfloor\frac{n}{2}\right\rfloor} c_{n-2 k}^{(n)} T_{n-2 k}(x) .
$$

Using these constants, we define a function

$$
u_{n}(x, y)=\sum_{k=0}^{\left\lfloor\frac{n}{2}\right\rfloor} c_{n-2 k}^{(n)} \varphi_{n-2 k}(x, y) .
$$

The function $u_{n}(x, y)$, being a linear combination of harmonic functions, is itself harmonic in the plane. Moreover $u_{n}(x, y)$ restricts to the function $x^{n}$ on the unit circle as seen from Equations (3.2) and (4.1). From the uniqueness of the solution to the Dirichlet problem, the function $u_{n}(x, y)$ is the sought for function of the theorem. Therefore it remains to determine the coefficients $c_{n-2 k}^{(n)}$. 
But these coefficients are well known, see for example [7, Equation (2.14)]. We know that for $n \geq 1$,

$$
c_{n-2 k}^{(n)}=\frac{1}{2^{n-1}}\left(\begin{array}{l}
n \\
k
\end{array}\right) \text { for } k=0, \ldots,\left\lfloor\frac{n}{2}\right\rfloor-1,
$$

and

$$
c_{n-2 k}^{(n)}=\frac{1}{2^{n-1}}\left(1-\frac{\epsilon_{n}}{2}\right)\left(\begin{array}{c}
n \\
\left\lfloor\frac{n}{2}\right\rfloor
\end{array}\right) \quad \text { for } k=\left\lfloor\frac{n}{2}\right\rfloor .
$$

Finally putting these coefficients into Equation (4.2) we obtain Equation (2.3), and a rearrangement of the terms gives Equation (2.1) of the theorem.

Next we determine the function $v_{n}(x, y)$.

As we did above, examining the nature of the Chebyshev polynomials of the second kind, we can find coefficients $d_{n-2 k}^{(n)}$ such that for all $n \geq 1$, we can write

$$
x^{n-1}=\sum_{k=0}^{\left\lfloor\frac{n}{2}\right\rfloor} d_{n-2 k}^{(n)} U_{n-2 k-1}(x) .
$$

Now we define a function $v_{n}(x, y)$ as

$$
v_{n}(x, y)=\sum_{k=0}^{\left\lfloor\frac{n}{2}\right\rfloor} d_{n-2 k}^{(n)} \psi_{n-2 k}(x, y) .
$$

Clearly, this function is harmonic and restricts to $x^{n-1} y$ on the unit circle for $n \geq 1$. It remains to determine the coefficients $d_{n-2 k}^{(n)}$. For this take the derivative of both sides of Equation (4.1) with respect to $x$, and use Equation (3.4) to get

$$
x^{n-1}=\sum_{k=0}^{\left\lfloor\frac{n}{2}\right\rfloor} \frac{n-2 k}{n} c_{n-2 k}^{(n)} U_{n-2 k-1}(x) .
$$

Comparing the coefficients of Equations (4.5) and (4.7), we find that, for $0 \leq$ $k \leq\left\lfloor\frac{n}{2}\right\rfloor$,

$$
d_{n-2 k}^{(n)}=\frac{n-2 k}{n} c_{n-2 k}^{(n)}=\frac{1}{2^{n-1}} \frac{n-2 k}{n}\left(\begin{array}{l}
n \\
k
\end{array}\right),
$$

where we used Equation (4.3) in the second equality. Note that we need not determine $d_{0}^{(n)}$ in case $n=2 k$, since in that case $U_{n-2 k-1}(x)=U_{-1}(x)=0$. Finally substituting the value of $d_{n-2 k}^{(n)}$ from Equation (4.8) into Equation (4.6) we get Equation (2.5) which in turn is equivalent to Equation (2.2). This then completes the proof of the theorem.

Remark. The apparent symmetry between Equations (2.1) and (2.2) seems to be lost between Equations (2.3) and (2.5). However the symmetry continues 
between them once we notice that for all $n \geq 1$ and $0 \leq k \leq\left\lfloor\frac{n}{2}\right\rfloor$,

$$
\begin{aligned}
\left(\begin{array}{l}
n \\
k
\end{array}\right) & =\left(\begin{array}{c}
n-1 \\
k
\end{array}\right)+\left(\begin{array}{l}
n-1 \\
k-1
\end{array}\right), \\
\frac{n-2 k}{n}\left(\begin{array}{l}
n \\
k
\end{array}\right) & =\left(\begin{array}{c}
n-1 \\
k
\end{array}\right)-\left(\begin{array}{l}
n-1 \\
k-1
\end{array}\right) .
\end{aligned}
$$

In particular

$$
u_{n}(x, y)=\frac{1}{2^{n-1}} \sum_{k=0}^{\left\lfloor\frac{n}{2}\right\rfloor},\left[\left(\begin{array}{c}
n-1 \\
k
\end{array}\right)+\left(\begin{array}{c}
n-1 \\
k-1
\end{array}\right)\right] \varphi_{n-2 k}(x, y),
$$

where $\sum^{\prime}$ means that the last term must be halved if $n$ is even, and

$$
v_{n}(x, y)=\frac{1}{2^{n-1}} \sum_{k=0}^{\left\lfloor\frac{n}{2}\right\rfloor}\left[\left(\begin{array}{c}
n-1 \\
k
\end{array}\right)-\left(\begin{array}{c}
n-1 \\
k-1
\end{array}\right)\right] \psi_{n-2 k}(x, y),
$$

where $n=1,2, \ldots$

\section{References}

[1] S. Axler, P. Bourdon, and W. Ramey, Harmonic Function Theory, Graduate Texts in Mathematics, Vol. 137, Second Edition, Springer-Verlag, New York, 2001.

[2] S. R. Bell, P. Ebenfelt, D. Khavinson, and H. S. Shapiro, On the classical Dirichlet problem in the plane with rational data, J. Anal. Math. 100 (2006), 157-190.

[3] _ Algebraicity in the Dirichlet problem in the plane with rational data, Complex Var. Elliptic Equ. 52 (2007), no. 2-3, 235-244.

[4] M. Chamberland and D. Siegel, Polynomial solutions to Dirichlet problems, Proc. Amer. Math. Soc. 129 (2001), no. 1, 211-217.

[5] P. Ebenfelt and M. Viscardi, An explicit solution to the Dirichlet problem with rational holomorphic data in terms of a Riemann mapping, Comput. Methods Funct. Theory 7 (2007), no. 1, 127-140.

[6] D. Khavinson and E. Lundberg, A tale of ellipsoids in potential theory, Amer. Math. Soc. 61 (2014), no. 2, 148-156.

[7] J. C. Mason and D. C. Handscomb, Chebyshev Polynomials, Chapman \& Hall/CRC, Boca Raton, FL, 2003.

[8] T. J. Rivlin, The Chebyshev Polynomials, Pure and Applied Mathematics, WileyInterscience [John Wiley \& Sons], New York, 1974.

Department of Mathematics

BiLKENT UNIVERSiTy

06800 Ankara, Turkey

E-mail address: sertoz@bilkent.edu.tr 